\title{
\begin{tabular}{|c|lc|}
\hline IV Simpósio Paranaense de Modelagem, & Artigo: & 09 \\
$\begin{array}{c}\text { Simulaçãoe Controle de Processos } \\
\text { ISSN : 1984-7521 }\end{array}$ & Páginas: & $\mathbf{6 2}$ - $\mathbf{6 6}$ \\
\cline { 2 - 2 } & & Pán
\end{tabular}
}

\section{EFEITO DO ULTRASSOM SOBRE O ESCOAMENTO GÁS- SÓLIDO NA REGIÃO DE ENTRADA DE UM RISER DE CFB}

\author{
Vivien Rossbach $^{* 1,2}$, Jonathan Utzig ${ }^{2}$, Natan Padoin ${ }^{1}$, Henry França Meier ${ }^{2}$, Cintia Soares ${ }^{1}$ \\ 1 - Universidade Federal de Santa Catarina-UFSC-SC, vivien.rossbach@posgrad.ufsc.br \\ 2 - Universidade de Blumenau - FURB, Blumenau - SC
}

\begin{abstract}
O perfil “core-annulus” encontrado em risers de leitos fluidizados circulantes dificulta o contato da fase sólida com a fase gasosa. O uso de ondas acústicas com frequência de $40 \mathrm{kHz}$ pode produzir forças radiais na direção contrária daquelas que fazem com que as partículas sólidas se concentrarem na parede e melhorar a sua dispersão ao longo da seção transversal do riser. Neste estudo foram analisados dois conjuntos com 20 transdutores de ultrassom, dispostos em 4 fileiras, variando o nível de pressão sonora (SPL), a posição axial do conjunto e o diâmetro dos transdutores. Os transdutores foram posicionados acima da entrada lateral de sólidos do riser. Os resultados das simulações com modelo euleriano mostram que nos dois casos o perfil "core-annulus" é suavizado e a dispersão de sólidos melhora ao longo da seção transversal do riser. A perda de pressão é reduzida devido à adição de energia acústica ao escoamento.
\end{abstract}

Palavras-chave: Leito fluidizado circulante (CFB). Correnteza acústica. Coeficiente de dispersão.

\section{Introdução}

Leitos fluidizados circulantes (CFB - Circulating Fluidized Beds) são utilizados em diferentes aplicações industriais, como o craqueamento catalítico fluido (FCC Fluid Catalytic Cracking) [1] e a decomposição de ozônio [2]. O perfil core-annulus, típico do escoamento gás-sólido ascendente em risers de CFB, caracteriza-se pela concentração de partículas nas paredes e a formação de uma região diluída no centro do duto, dificultando o contato da fase sólida com a fase gasosa. Diferentes alternativas foram propostas para melhorar a dispersão de partículas, como o uso de defletores aerodinâmicos na região de entrada de sólidos no riser $[3,4]$. Considerando a existência de forças radiais que levam as partículas a se concentrarem na parede do duto [5], o uso de ondas acústicas pode ser uma alternativa viável para melhorar a dispersão de partículas na seção transversal do riser. Como a velocidade radial do escoamento é muito menor que a velocidade axial [3], não é necessária uma força acústica tão grande para vencer as forças radiais como a que seria necessária para vencer forças axiais, que contam com a ação da gravidade. No entanto, é necessário utilizar ondas de ultrassom, cuja frequência é suficiente para capturar partículas na ordem de micrometros que são utilizadas nas aplicações de CFB. Há diferentes modelos matemáticos usados para descrever a ação de ondas de ultrassom em meio gasoso, os quais podem se basear na teoria da radiação acústica [6,7] ou de correnteza acústica [8]. No entanto, modelos de correnteza acústica podem descrever melhor a atuação do ultrassom em um meio turbulento $[8,10]$.

O objetivo deste estudo é verificar se a aplicação de ondas acústicas com frequência de $40 \mathrm{kHz}$ é suficiente para reduzir a formação do perfil core-annulus e melhorar a distribuição de partículas na seção transversal do riser. Para isto foram analisadas através de simulação computacional duas configurações geométricas com 20 transdutores de ultrassom instaladas na região de entrada do riser e o coeficiente de dispersão de partículas foi comparado com e sem onda acústicas. 


\section{Metodologia e Modelagem Matemática}

$\mathrm{O}$ riser de CFB estudado tem $104 \mathrm{~mm}$ de diâmetro interno e $3 \mathrm{~m}$ de altura. Ar ambiente, considerado como gás ideal, é alimentado na base com velocidade de 5,6 m/s e esferas de vidro, com diâmetro médio de $72 \mu \mathrm{m}$ e massa específica igual a 2500 $\mathrm{kg} / \mathrm{m}^{3}$, são alimentadas em uma entrada lateral com $45^{\circ}$ de inclinação em relação à vertical, com razão de carga de $25,7 \mathrm{~kg}$ de sólidos por $\mathrm{kg}$ de gás. Para simular o escoamento gás-sólido com e sem ondas acústicas, foi utilizado o modelo eulerianoeuleriano gás-sólido. A turbulência da fase gasosa foi modelada utilizando o modelo kepsilon, as propriedades da fase sólida foram descritas através da teoria cinética do escoamento granular (KTGF) e o arraste foi representado pelo modelo de Gidaspow, conforme descrito em Rossbach et al. [11]. A correnteza acústica produzida foi representada pelo modelo proposto por Sajjadi et al. [9] (Eq. 1), que foi implementado como condição de entrada de pressão nas superfícies dos transdutores para produzir ondas mecânicas análogas às ondas acústicas [12]:

$$
P(t)=-P_{a} \sin (\omega t)
$$

A pressão acústica é dada por:

$$
\mathrm{P}_{\mathrm{a}}=\sqrt{2 \rho_{\mathrm{g}} \mathrm{I}_{\mathrm{US}} \mathrm{c}_{0}}
$$

Nas equações 1 e 2, $\omega$ é a frequência angular ( $\mathrm{rad} / \mathrm{s}), \rho_{\mathrm{g}}$ é a massa específica da fase gasosa, igual a $1,2 \mathrm{~kg} / \mathrm{m}^{3}$ e $\mathrm{c}_{0}$ é a velocidade de fase da onda acústica no ar, igual a $346 \mathrm{~m} / \mathrm{s}$. As ondas acústicas são emitidas por um conjunto de transdutores de ultrassom com frequência de $40 \mathrm{kHz}$ dispostos em dois arranjos diferentes, como indicado na Tabela 1. Os transdutores têm diâmetro de $16 \mathrm{~mm}$ no Caso 1 e $10 \mathrm{~mm}$ no Caso 2. Nos dois casos são utilizados 20 transdutores dispostos em quatro fileiras de cinco transdutores, sendo que duas fileiras são paralelas à entrada de sólidos e as outras duas são perpendiculares. A posição axial do conjunto de transdutores é de $50 \mathrm{~mm}$ acima da entrada de ar para o Caso 1 e $70 \mathrm{~mm}$ para o Caso 2. O nível de pressão sonora (SPL) emitida é de $120 \mathrm{~dB}$ para o Caso 1 e $90 \mathrm{~dB}$ para o Caso 2.

Para capturar o campo acústico produzido pelo ultrassom, é necessário que as células da malha numérica tenham dimensões menores que o comprimento da onda e que o passo de tempo adotado seja menor que o inverso do dobro da frequência [7]. Neste caso, os elementos da malha devem ser menores que $0,00785 \mathrm{~m}$ e o passo de tempo deve ser menor que $1,25 \times 10^{-6}$. As malhas numéricas utilizadas têm 798000 elementos e foram refinadas na região dos transdutores para atingir este critério. No entanto, para as simulações seguintes é necessário obter $5 \mathrm{~s}$ de média transiente, o que se torna impraticável com um passo de tempo muito pequeno. Como não se deseja obter o campo acústico, mas sim comparar o seu efeito sobre o escoamento com diferentes arranjos, utilizou-se um passo de tempo de $1 \times 10^{-4} \mathrm{~s}$, com critério de convergência igual a $10^{-4}$ para os resíduos.

\section{Resultados e Discussão}

Na Tabela 1 são apresentados o coeficiente de dispersão médio de partículas sólidas na seção transversal $\left(\mathrm{C}_{\mathrm{v}}\right)$, a perda de carga global no riser $(\Delta \mathrm{P})$ e o inventário global médio de sólidos. $O$ coeficiente de dispersão é calculado como sendo a razão entre o desvio-padrão e a média da fração volumétrica das seções transversais a $55 \mathrm{~mm}$, $80 \mathrm{~mm}$ e $100 \mathrm{~mm}$ de altura em relação à base do riser. O uso de ondas acústicas leva a 
uma redução no coeficiente de dispersão de $42 \%$ para o Caso 1 e $49 \%$ para o Caso 2 frente ao caso sem sua utilização. As ondas acústicas aplicadas na direção radial produzem uma força na direção contrária às forças radiais que levam as partículas para a parede [5], melhorando a distribuição de partículas e aumentando sua concentração próximo ao centro do riser. A perda de carga se reduz em $22 \%$ no Caso 1 e em $29 \%$ no Caso 2 em relação à simulação sem ondas acústicas. Isto ocorre porque a energia acústica é somada à energia do escoamento e compensa perdas de carga por outros fatores. O inventário de sólidos no riser se reduz em $22 \%$ no Caso 1 e $18 \%$ no Caso 2 devido à aceleração das partículas causada quando estas passam pelos transdutores. A diminuição do inventário e a redução da perda de carga possibilitam trabalhar com razões carga maiores [4].

Tabela 1: Propriedades geométricas e resultados obtidos com e sem ondas acústicas.

\begin{tabular}{lllllll}
\hline Caso & $\begin{array}{l}\text { Diâmetro } \\
\text { do } \\
\text { transdutor } \\
{[\mathrm{mm}]}\end{array}$ & $\begin{array}{l}\text { Posição } \\
\text { axial do } \\
\text { conjunto de } \\
\text { transdutores } \\
{[\mathrm{mm}]}\end{array}$ & $\mathrm{SPL}[\mathrm{dB}]$ & $\mathrm{C}_{\mathrm{v}}[-]$ & $\Delta \mathrm{P}[\mathrm{Pa}]$ & $\begin{array}{l}\text { Inventário } \\
\text { médio de } \\
\text { sólidos }[\mathrm{g}]\end{array}$ \\
\hline $\begin{array}{l}\text { Sem ondas } \\
\text { acústicas } \\
\text { (COP 1) }\end{array}$ & - & - & - & 1,650 & 44,7 & 16,5 \\
1 & 16 & 50 & 120 & 0,949 & 34,9 & 13,2 \\
2 & 10 & 70 & 90 & 0,839 & 31,5 & 13,5 \\
\hline
\end{tabular}
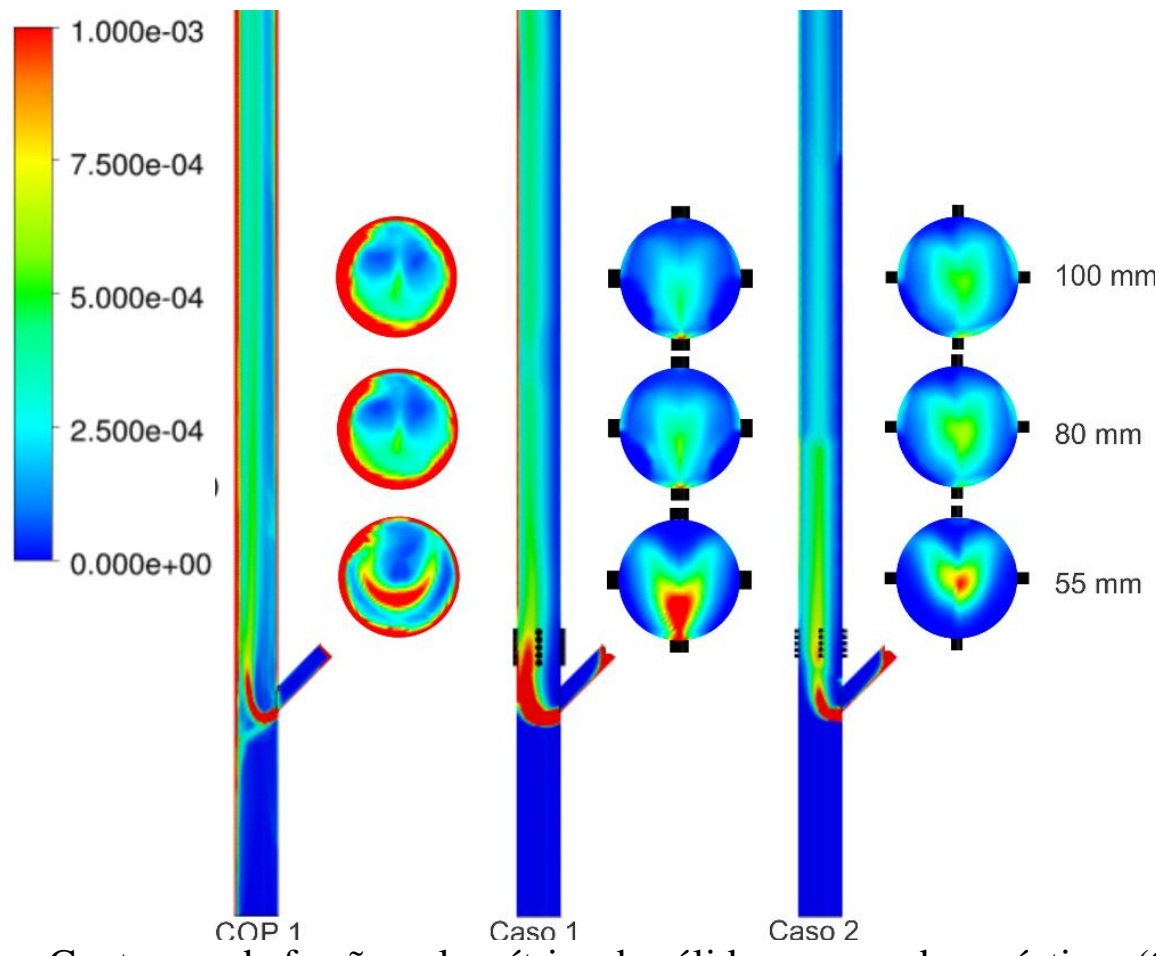

Figura 1 - Contornos de fração volumétrica de sólidos com ondas acústicas (Caso 1 e Caso 2) e sem ondas acústicas (COP 1).

Na Figura 1 são apresentados os contornos de fração volumétrica para os casos sem ondas acústicas (COP 1) e com ondas acústicas (casos 1 e 2). No caso sem ondas acústicas há a formação do perfil core-annulus em toda a extensão do riser, porém a concentração de partículas é maior na parede oposta à entrada de sólidos. Nos casos $1 \mathrm{e}$ 2 o perfil core-annulus é quebrado devido ao efeito das ondas acústicas. O Caso 2 
apresenta melhores resultados apesar de utilizar transdutores com menor diâmetro, que emitem menor quantidade de energia por metro quadrado de superfície.
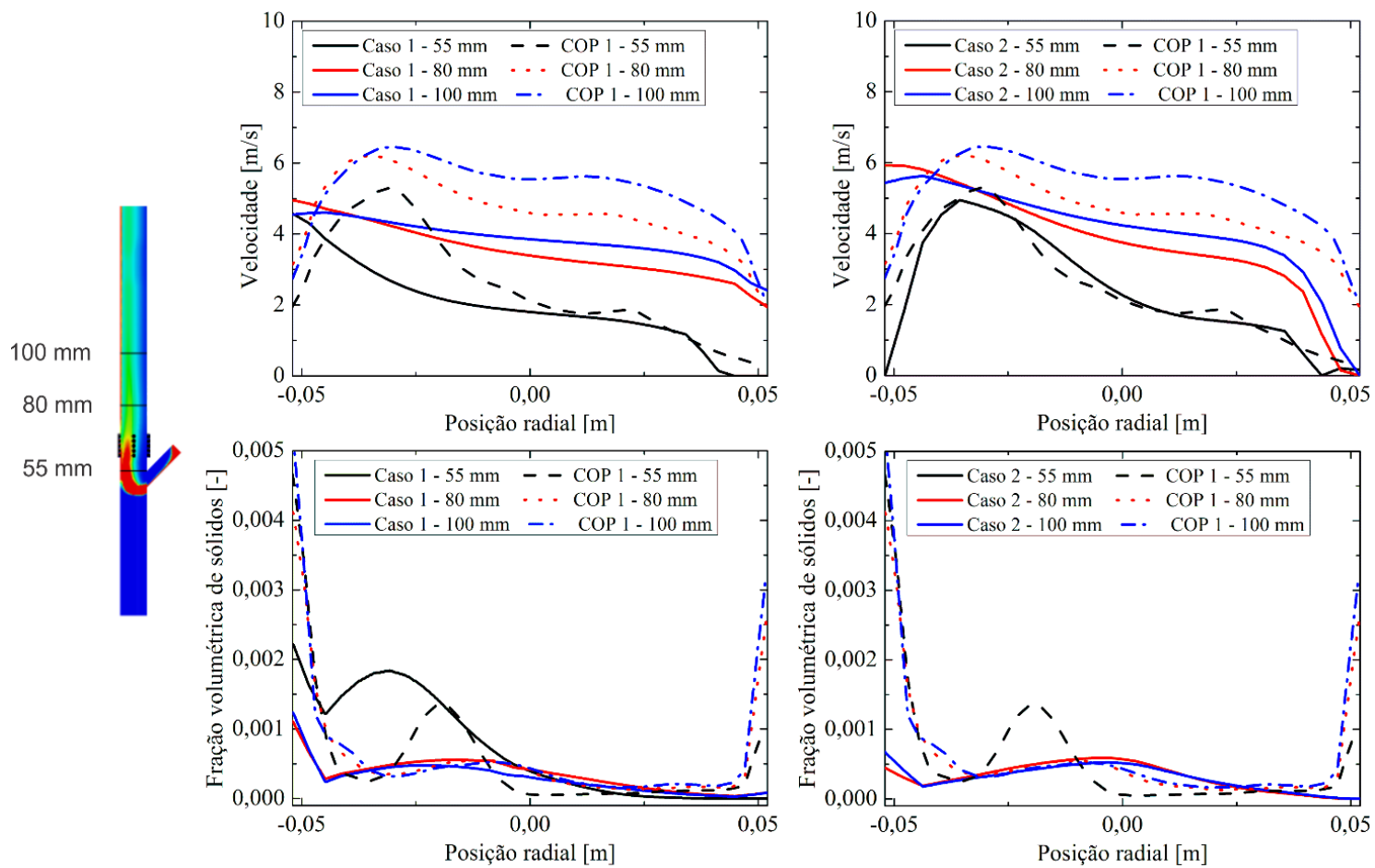

Figura 2 - Perfis de velocidade e fração volumétrica da fase sólida com e sem ondas acústicas.

A energia acústica aplicada no Caso 1 é maior que a necessária para dispersar as partículas sólidas e faz com que estas se concentrem no centro do riser. A dispersão de partículas é mais homogênea para os Casos 1 e 2 na região acima dos transdutores ( 80 $\mathrm{mm})$ e se mantém com a altura.

A Figura 2 mostra os perfis de velocidade e fração volumétrica da fase sólida para os Casos 1 e 2 em comparação com o caso sem ondas acústicas (COP 1). Nos dois casos há um aumento de velocidade na parede e a sua suavização no centro do riser, porém no Caso 2 a velocidade da fase sólida sem mantém baixa em uma das paredes. Observa-se também uma grande redução da fração volumétrica de sólidos na parede com o uso de ondas acústicas para os dois casos apresentados.

\section{Conclusão}

$\mathrm{O}$ uso de transdutores de ultrassom mostrou-se uma alternativa viável para melhorar a dispersão de partículas sólidas em risers de CFB e reduzir a formação do perfil core-annulus. As duas configurações propostas atingiram os resultados esperados, ocasionando a diminuição da fração volumétrica de sólidos nas paredes do riser e o aumento da dispersão de sólidos, medido através do coeficiente de dispersão.

\section{Agradecimentos}

Este estudo foi financiado em parte pela Coordenação de Aperfeiçoamento de Pessoal de Nível Superior - Brasil (CAPES) - Código Financeiro 001. Os autores agradecem o apoio financeiro da Petróleo Brasileiro S.A. (Petrobras), através dos termos de cooperação 0050.0070 334.11.9 e 5850.0103010.16.9.

\section{Referências}

[1] G.C. Lopes; L.M. Rosa; M. Mori; J.R. Nunhez; W.P. Martignoni. Threedimensional modeling of fluid catalytic cracking industrial riser flow and reactions. Comput. Chem. Eng. 2011. 35. 2159-2168. 
[2] P. Jiang; H. Bi; R.H. Jean; L.S. Fan. Baffle effects on performance of catalytic circulating fluidized bed reactor. AIChE Journal, 1991. 37. 1392-1400.

[3] V. Rossbach; J. Utzig; R.K. Decker; H.F. Meier. Experimental analysis of the gas-solid flow in a ring-baffled CFB riser using Laser Doppler Anemometry ( LDA ) and Phase-Doppler Anemomety ( PDA ). in: Proc. EPTT 2016 - 10th ABCM Spring Sch. Transit. Turbul., São José dos Campos - SP, Brazil, 2016.

[4] V. Rossbach; J. Utzig; R.K. Decker; D. Noriler; C. Soares; W.P. Martignoni; H.F. Meier. Gas-solid flow in a ring-baffled CFB riser: Numerical and experimental analysis, Powder Technology. 2019.

[5] Y. Fan; C. Li; H. Fan; M. Yu; C. Lu. Core-Annulus radial solids concentration distribution in riser. Chemical Engineering Science, 2018. 192. 318-334.

[6] M.A.B. Andrade; N. Perez; J.C. Adamowski. Analysis of a non-resonant ultrasonic levitation device. in: Phys. Procedia. 2015. pp. 68-71.

[7] C. Knoop; U. Fritsching. Gas/particle interaction in ultrasound agitated gas flow. Procedia Eng. 2012. 42. 770-781.

[8] N. Riley. Acoustic Streaming, Theor. Comput. Fluid Dyn. 1998. 10. 349-356.

[9] B. Sajjadi; A.A.A. Raman; S. Ibrahim. Influence of ultrasound power on acoustic streaming and micro-bubbles formations in a low frequency sono-reactor: Mathematical and 3D computational simulation, Ultrason. Sonochem. 2015. 24.

[10] M. Červenka; M. Bednařík. Variety of acoustic streaming in 2D resonant channels. Wave Motion. 2016. 66. 21-30.

[11] V. Rossbach; J. Utzig; R.K. Decker; D. Noriler; H.F. Meier. Numerical gas-solid flow analysis of ring-baffled risers. Powder Technology. (2016).

[12] V. Rossbach; N. Padoin; J. Utzig; H.F. Meier; C. Soares. Influência da Correnteza Acústica sobre a Dispersão de Partículas em um Riser De CFB. in: Blucher Chem. Eng. Proc., Editora Blucher, São Paulo, 2018: pp. 818-821. 